\title{
MicroRNA-339-5p inhibits colorectal tumorigenesis through regulation of the MDM2/p53 signaling
}

\author{
Cen Zhang ${ }^{1, *}$, Juan Liu ${ }^{1, *}$, Xiaolong Wang ${ }^{1,2}$, Rui Wu ${ }^{1}$, Meihua Lin ${ }^{1}$, Saurabh V. \\ Laddha ${ }^{3}$, Qifeng Yang ${ }^{1,2}$, Chang S. Chan ${ }^{3}$ and Zhaohui Feng ${ }^{1}$ \\ ${ }^{1}$ Department of Radiation Oncology, Rutgers Cancer Institute of New Jersey, Rutgers, State University of New Jersey, New \\ Brunswick, NJ, USA \\ ${ }^{2}$ Department of Breast Surgery, Qilu Hospital, Shangdong University, Ji'nan, China \\ ${ }^{3}$ Center for Systems Biology, Rutgers Cancer Institute of New Jersey, Rutgers, State University of New Jersey, New Brunswick, \\ NJ, USA \\ * These auhors contributed equally to this work \\ Correspondence to: Zhaohui Feng, email: fengzh@cinj.rutgers.edu \\ Keywords: microRNA-339-5p; colorectal cancer; p53; migration; invasion; tumorigenesis \\ Received: August 04, $2014 \quad$ Accepted: August 20, $2014 \quad$ Published: August 21, 2014
}

This is an open-access article distributed under the terms of the Creative Commons Attribution License, which permits unrestricted use, distribution, and reproduction in any medium, provided the original author and source are credited.

\section{ABSTRACT}

Tumor suppressor p53 plays a central role in tumor suppression. To ensure its proper function, the levels and activity of p53 are under a tight regulation in cells. MicroRNAs are short non-coding RNAs that play an important role in regulation of gene expression. Recently, microRNA-339-5p has been reported to be frequently downregulated in colorectal cancer, and furthermore, its down-regulation is associated with poor prognosis in cancer patients, which strongly suggests a tumor suppressive function of microRNA-339-5p in colorectal cancer. In this study, we found that microRNA-339-5p directly represses the expression of MDM2, a key negative regulator of p53, through binding to MDM2 3'-UTR in colorectal cancer cells. Through the downregulation of MDM2, microRNA-339-5p increases p53 protein levels and functions, including p53 transcriptional activity and p53-mediated apoptosis and senescence in response to stress. Furthermore, microRNA-339-5p inhibits the migration and invasion of colorectal cancer cells and the growth of colorectal xenograft tumors in a largely p53-dependent manner. Our results highlighted an important role of microRNA-339$5 p$ in suppression of colorectal tumorigenesis, and also revealed that regulating the p53 function is an important mechanism for microRNA-339-5p in tumor suppression.

\section{INTRODUCTION}

The tumor suppressor p53 and its signaling pathway play a central role in suppression of tumorigenesis, including colorectal cancer [1-3]. In response to stress, p53 is activated and accumulated in cells, which in turn transcriptionally regulates many target genes to initiate various cellular responses to maintain genomic stability and prevent tumorigenesis, including apoptosis, cell cycle arrest and senescence [1-3]. p53 also plays a critical role in inhibition of migration, invasion and metastasis of cancer cells $[1,2,4]$. To ensure its proper function in tumor suppression, the levels and activities of p53 are under a tight and complex regulation by many different regulators and mechanisms in cells $[2,5,6]$. MDM2, an
E3 ubiquitin ligase, is the most critical negative regulator for $\mathrm{p} 53$. MDM2 binds to $\mathrm{p} 53$ and degrades $\mathrm{p} 53$ through ubiquitination to keep the p53 at a low level under the non-stress conditions [7, 8]. Importantly, MDM2 itself is a transcription target of p53. Thus, p53 and MDM2 form an auto-regulatory negative feedback loop, which is tightly controlled to allow the appropriate response of $\mathrm{p} 53$ to stress in cells [5, 6]. MDM2 is frequently overexpressed in various types of tumors, including colorectal cancer, which attenuates $\mathrm{p} 53$ function and promotes tumorigenesis [9-11] .

MicroRNAs (miRNAs) are a class of endogenously expressed, small (20-25 nucleotides) non-coding regulatory RNA molecules in cells. MiRNAs bind to the 3'-untranslated regions (3'- UTRs) of mRNAs in a 
sequence-specific manner, reducing mRNA stability and inhibiting translation, and thereby negatively regulate the expression of genes at the posttranscriptional level [1214]. Recently, emerging evidence has shown that miRNAs are an important component of the p53 signaling pathway in addition to protein-coding genes [15-17]. p53 positively or negatively regulates the expression of certain miRNAs, which in turn mediates p53 function. At the same time, miRNAs also regulate p53 levels and functions through regulating p53 or regulators for p53 in cells. For instance, miR-125b and miR-504 have been recently identified as the direct negative regulators of $\mathrm{p} 53$ [15, 18, 19]. miR192, miR-194, miR-25, miR-32 and miR-661 have been reported to directly repress MDM2 expression to activate p53 in cells [20-22]. These findings demonstrate that as a new component of the p53 signaling pathway, miRNAs play an important role in regulating and/or mediating p53 function in tumor suppression. The expression of many miRNAs is frequently altered in tumors [23, 24]. The altered expression of certain miRNAs could lead to the disruption of the fine balance between miRNAs and the p53 signaling pathway, and thus attenuates p53 function and contributes to tumorigenesis $[15,17]$.

Recently, microRNA-339-5p (miR-339-5p) was reported to be frequently down-regulated in different types of cancers, including colorectal cancers, which is associated with cancer metastasis and poor prognosis in cancer patients [25-27]. In this study, we found that miR339-5p directly represses MDM2 expression, which in turn increases p53 protein levels and enhances p53 functions in regulating apoptosis and senescence in colorectal cancer cells in response to stress. Furthermore, miR-339-5p inhibits the migration and invasion of colorectal cancer cells as well as the growth of colorectal xenograft tumors in mice largely through the up-regulation of p53 functions. These results demonstrated that the up-regulation of p53 function is an important mechanism by which miR-339-5p inhibits colorectal tumorigenesis.

\section{RESULTS}

\section{MiR-339-5p down-regulates MDM2 expression through its binding to the 3'-UTR of MDM2}

Recently, miRNA-339-5p was reported to be frequently down-regulated in colorectal cancer, and furthermore, its decreased expression is associated with poor prognosis in colorectal cancer patients, which strongly suggests a potential role of miR-339-5p in suppression of colorectal cancer [25]. However, its underlying mechanism is not well-understood. To further understand the mechanism by which miR-339-5p exhibits tumor suppressive function in colorectal cancer, we performed a computational search for the potential targets for miR-339-5p. MDM2 was identified as a potential target for miR-339-5p, which contains multiple putative binding sites for miR-339-5p in its 3'-UTR.

To examine whether miR-339-5p can regulate MDM2 protein levels, human colorectal cancer HCT116 p53+/+, HCT116 p53-/-, RKO p53+/+ and RKO p53-/- cells were transfected with miR-339-5p mimic or scrambled miRNA control (miR-con). These cell lines are two pairs of isogenic human colorectal cell lines with or without expression of wild-type (WT) p53, which have been widely used for p53 study [28-31]. MiR-339-5p mimic greatly reduced MDM2 protein levels in all these four colorectal cancer cell lines (Figure 1A). As a key negative regulator for $\mathrm{p} 53$, the down-regulation of MDM2 by miR-339-5p resulted in the induction of p53 protein in HCT116 p53+/+ and RKO p53+/+ cells. Furthermore, miR-339-5p mimic also reduced MDM2 mRNA levels in these cell lines, but to a less extent than its effect on MDM2 protein levels (Figure 1B). Interestingly, a stronger inhibitory effect of miR-339-5p on both MDM2 protein and mRNA was observed in HCT116 p53-/- and RKO p53-/- cells compared with HCT116 p53+/+ and RKO p53+/+ cells (Figure 1A,B). Since MDM2 is a direct transcriptional target of $\mathrm{p} 53$, the down-regulation of MDM2 by miR-339-5p could increase p53 protein levels and in turn transcriptionally induce MDM2, which could partially compromise the inhibitory effect of miR-339-5p on MDM2. In addition to the above-mentioned cell lines, the repression of MDM2 by miR-339-5p and the resultant induction of p53 protein levels were also observed in other cell lines, including human lung H460 cells and breast MCF7 cells which express WT p53 (Figure 1C), indicating that miR-339-5p represses MDM2 expression in different types of cells. To further confirm that miR339-5p represses MDM2, the miR-339-5p inhibitor, single-stranded RNA oligonucleotides that completely match with mature miR-339-5p sequences, was used to treat HCT116 p53+/+, HCT116 p53-/-, RKO p53+/+ and RKO p53-/- cells. As shown in Figure 1D, the miR$339-5 \mathrm{p}$ inhibitor increased the MDM2 protein levels in all these cell lines, and reduced p53 protein levels in HCT116 p53+/+ and RKO p53+/+ cells. Taken together, these results demonstrated that miR-339-5p can negatively regulate MDM2 in cells.

Computational analysis predicted three putative binding sites for miR-339-5p located in the first $1.6 \mathrm{~kb}$ of 3'-UTR of the human MDM2 mRNA (Figure 1E). To determine whether miR-339-5p binds to these three sites to down-regulate MDM2, a firefly luciferase reporter vector was constructed by inserting the first $1.6 \mathrm{~kb}$ of the 3'-UTR cDNA sequences of the human MDM2 gene containing these three sites into the 3 ' end of the luciferase gene. The vector was transfected into HCT116 p53+/+ or RKO p53+/+ cells together with either miR-339-5p mimic or miR-con. Compared with miR-con, miR-3395 p significantly decreased (by $\sim 2-2.5$-fold) the luciferase 
A

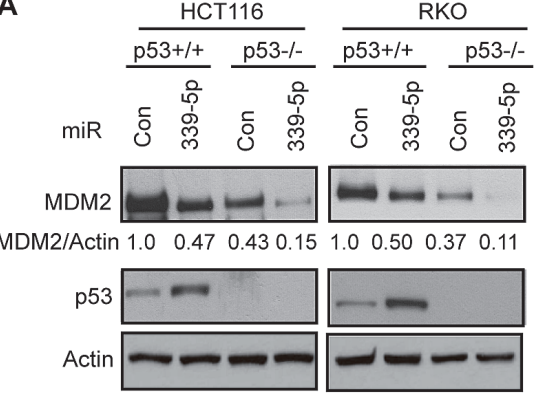

B $\square$ miR-con

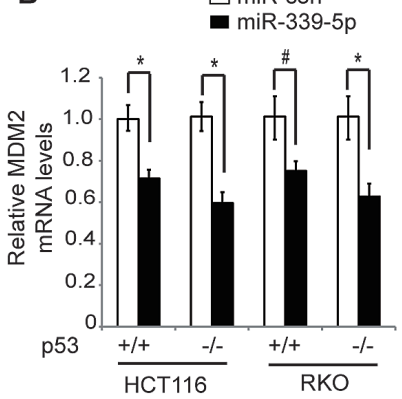

C

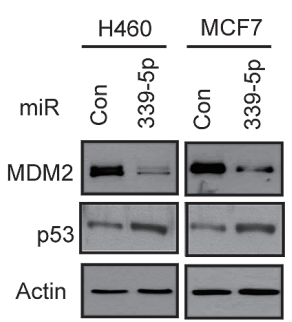

D

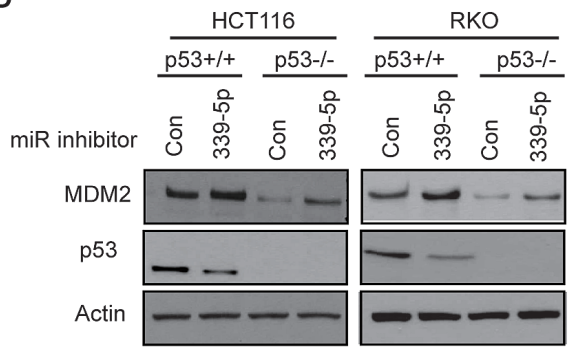

E

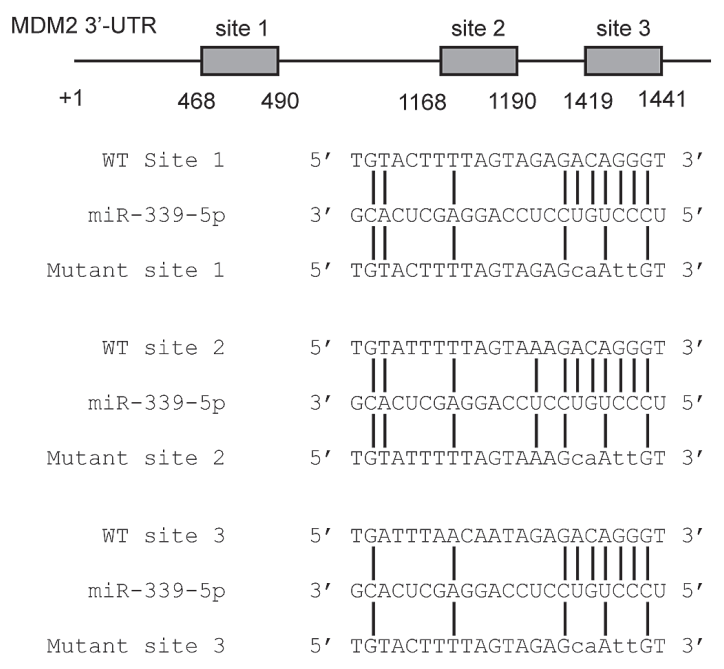

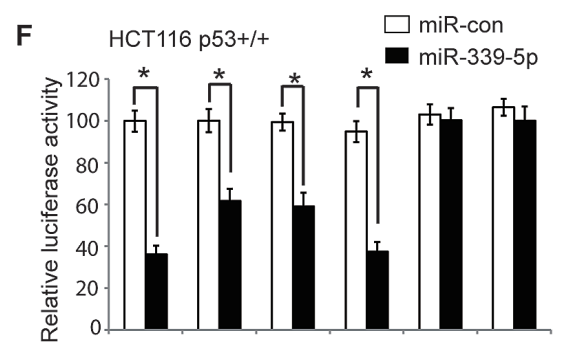

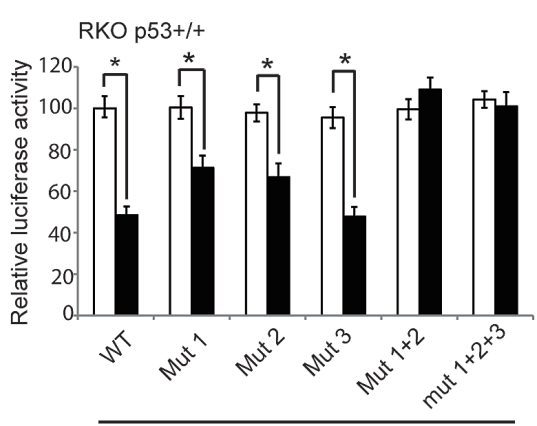

MDM2 3'-UTR

Figure 1: MiR-339-5p negatively regulates MDM2 levels in human colorectal cells through binding to human $M D M 2$ 3'-UTR. (A) MiR-339-5p decreased MDM2 protein levels and increased p53 protein levels in human colorectal cancer HCT116 and RKO cells. HCT116 p53+/+, HCT116 p53-/-, RKO p53+/+ and RKO p53-/- cells were transfected with miR-339-5p mimic or scrambled miRNA control (miR-con), and the MDM2 and p53 protein levels were measured at $24 \mathrm{~h}$ after transfection by western-blot assays. (B) MiR-339-5p decreased MDM2 mRNA levels in HCT116 and RKO cells. The MDM2 mRNA levels were measured by Taqman real-time PCR in cells transfected with miR-339-5p mimic or miR-con, and normalized with actin. The levels of the MDM2 mRNA in control cells transfected with miR-con were designated as 1 . Data are presented as mean $\pm \mathrm{SD}(\mathrm{n}=3) . \#: p<0.05, *: p<0.01$, two-tailed Student $t$-tests. (C) MiR-339-5p decreased MDM2 protein levels and increased p53 protein levels in human lung H460 and breast MCF7 cells. (D) The miR-339-5p inhibitor increased MDM2 protein levels and reduced p53 protein levels in HCT116 and RKO cells. Cells were transfected with the miR-339-5p inhibitor or miR-con inhibitor, and the MDM2 and p53 protein levels were measured at $24 \mathrm{~h}$ after transfection by western-blot assays. (E) The sequences of miR-339-5p, its putative binding sites and their mutants in the 3'-UTR of human MDM2. The positions of putative binding sites are labeled. The drawing is not to scale. (F) miR-339-5p inhibited the luciferase activities of the luciferase reporter vectors containing the wild-type (WT) human MDM2 3'-UTR which includes the 3 putative binding sites. HCT116 p53+/+ and RKO p53+/+ cells were transfected with luciferase reporter vectors containing WT or different mutant human MDM2 3'-UTR together with miR-339-5p mimic or miR-con. Luciferase activities were measured at $24 \mathrm{~h}$ after transfection. Data are presented as mean $\pm \mathrm{SD}(\mathrm{n}=$ 3). *: $p<0.01$, two-tailed Student $t$-tests. 
activities of the vectors containing the WT MDM2 3'UTR sequences in both HCT116 p53+/+ and RKO p53+/+ cells (Figure 1F). We further constructed serial luciferase vectors containing the mutant MDM2 3'-UTR sequence by mutating different putative binding sites for miR339-5p. As shown in Figure 1F, mutating either putative binding site 1 (Mut 1) or site 2 (Mut 2) partially rescued the luciferase activities reduced by miR-339-5p, whereas mutating the putative binding site 3 (Mut 3) alone failed to do so. Consistently, mutating the putative binding sites 1 and 2 together (Mut 1+2) almost completely abolished the inhibitory effect of miR-339-5p on the luciferase activities, whereas mutating these 3 putative binding sites together (Mut 1+2+3) did not further increase the luciferase activities. These results indicated that miR-339$5 \mathrm{p}$ targets $M D M 2$ through direct binding to the first two binding sites in MDM2 3'-UTR.

A

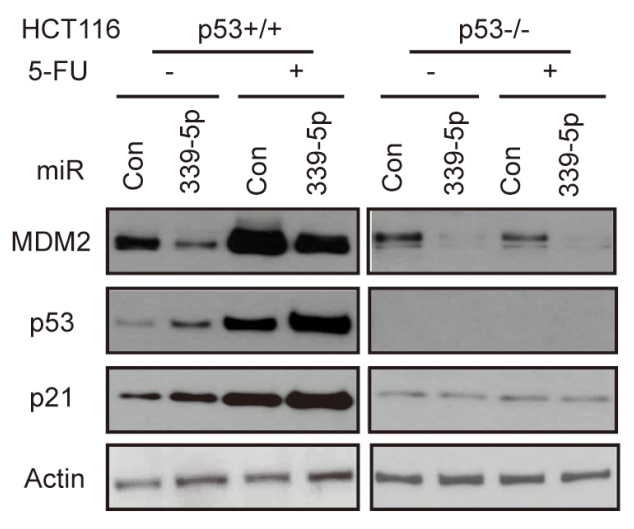

C

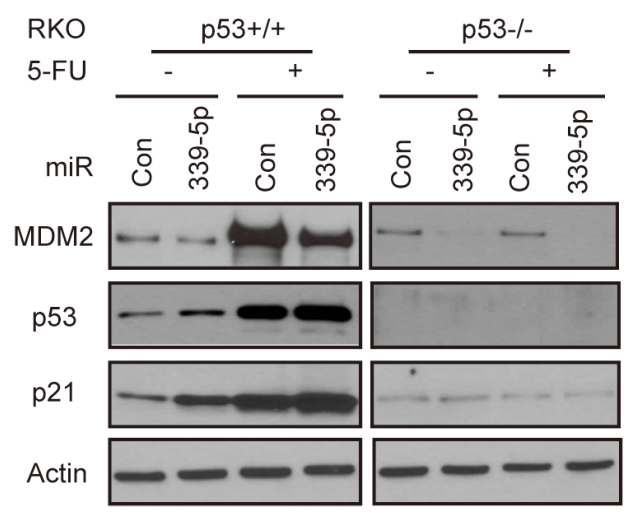

MiR-339-5p increases $p 53$ protein accumulation and its transcriptional activity in response to stress

p53 protein is maintained at a low level in cells under the non-stressed conditions mainly through proteasomal degradation of p53 protein by E3 ubiquitin ligases, particularly MDM2 [5, 6, 32]. In response to stress signals, p53 protein is accumulated in cells, which in turn leads to the transcriptional activation of p53 target genes to exert p53 functions in tumor suppression [1-3]. To investigate whether miR-339-5p reduces the $\mathrm{p} 53$ protein accumulation and p53 transcriptional activity in response to stress in colorectal cancer cells, HCT116 p53+/+ and HCT116 p53-/- cells transfected with miR-339-5p mimic were treated with chemotherapeutic agent 5-Fluorouracil (5-FU), which acted as stress signal to activate p53. $5-\mathrm{FU}$ is the most widely-used chemotherapeutic agent

B
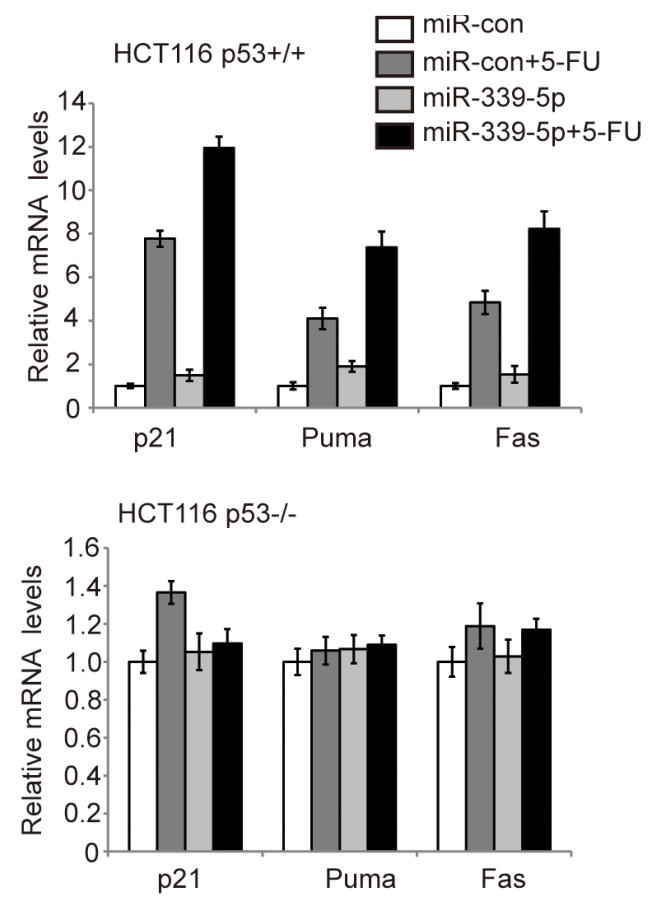

Figure 2: MiR-339-5p increases p53 protein accumulation and its transcriptional activity in response to stress by negatively regulating MDM2 in human colorectal cancer cells. (A) MiR-339-5p decreased MDM2 protein levels and increased the p53 protein accumulation and transcriptional activity toward p21 in response to stress in HCT116 cells. (B) MiR-339-5p increased the p53 transcriptional activity toward p21, Puma and Fas in response to stress in HCT116 cells. In A and B: HCT116 p53+/+ and p53-/- cells were transfected with miR-339-5p mimic or miR-con. At $24 \mathrm{~h}$ after transfection, cells were treated with 5-FU (50 $\mu \mathrm{M})$ for $8 \mathrm{~h}$, and analyzed by western-blot (A) and Taqman real-time PCR (B), respectively. The mRNA levels of all genes were normalized to actin. The mRNA levels of genes in untreated cells transfected with miR-con were designated as 1 . Data are presented as mean \pm SD $(n=3)$. (C) MiR-339-5p decreased MDM2 protein levels and increased p53, p21 protein levels in response to stress in RKO cells. RKO p53+/+ and RKO p53-/cells were treated with 5-FU and analyzed as described in A. 
for colorectal cancer $[33,34]$. The miR-339-5p mimic induced p53 protein levels in HCT116 p53+/+ cells under both non-stressed and stressed conditions (5-FU treatment) (Figure 2A). Consistent with the increased p53 protein accumulation, the levels of p21 protein, a well-known p53 target, was also increased by miR-339-5p under both non-stressed and stressed conditions in a p53-dependent manner; p21 was clearly induced by miR-339-5p in p53+/+ but not p53-/- HCT116 cells (Figure 2A). This result demonstrated that the induction of $\mathrm{p} 21$ by miR-339$5 p$ is due to the activation of p53 through MDM2 downregulation. The increased p53 transcriptional activity by miR-339-5p was also confirmed by examining the mRNA levels of several p53 target genes, including p21 (involved in cell cycle and senescence), Puma and Fas (involved in apoptosis). MiR-339-5p induced the mRNA levels of these genes in HCT116 p53+/+ cells with or without 5-FU treatment as detected by real-time PCR assays (Figure $2 \mathrm{~B}$, upper panel). Furthermore, the mRNA levels of these genes were not affected by miR-339-5p in HCT116 p53/- cells treated with or without 5-FU (Figure 2B, lower panel). In addition to HCT116 cells, miR-339-5p mimic reduced the MDM2 protein levels, which in turn increased the protein levels of p53 and p21, in RKO p53+/+ cells treated with or without 5-FU (Figure 2C). These results together showed that miR-339-5p enhances p53 protein accumulation and p53 transcriptional activity in response to stress in colorectal cancer cells.

\section{MiR-339-5p enhances p53 functions in mediating apoptosis and senescence in response to stress}

The p53-mediated apoptosis and senescence are the main mechanisms for $\mathrm{p} 53$ in tumor suppression $[1,2]$. To investigate the impact of miR-339-5p upon p53-mediated apoptosis, HCT116 p53+/+ and p53-/- cells transfected with miR-339-5p mimic were treated with 5-FU, and cellular apoptosis was measured in a flow cytometer. It has been reported that 5-FU induces apoptosis in a largely p53-dependent manner in colorectal cancer cells, which is critical for colorectal cancer to respond to 5-FU-based chemotherapy $[33,34]$. As shown in Figure 3A, the apoptosis induced by 5-FU was highly p53-dependent; much more cells underwent apoptosis in HCT116 p53+/+ cells than HCT116 p53-/- cells. Notably, the miR-339-5p mimic singificantly promoted 5 -FU-induced apoptosis in HCT116 p53+/+ cells. In contrast, much less pronounced effect of miR-339-5p on 5-FU-induced apoptosis was observed in HCT116 p53-/- cells. Furthermore, the miR339-5p inhibitor significantly decreased 5-FU-induced apoptosis in HCT116 p53+/+ cells but not in HCT116 p53-/- cells (Figure 3B). These results demonstrated that miR-339-5p enhances $\mathrm{p} 53$ function in mediating apoptosis in colorectal cells in response to stress.

It has been reported that chemotherapeutic agent
Doxorubicin induces senescence in a largely p53dependent manner in many different types of cell lines $[35,36]$. To investigate whether miR-339-5p affects p53 function in inducing senescence, HCT116 p53+/+ and p53-/- cells transfected with the miR-339-5p mimic were treated with Doxorubicin, and senescent cells were detected by senescence associated $\beta$-galactosidase (SA$\beta$-gal) staining. Doxorubicin induced senescence in a largely p53-dependent manner in HCT116 cells (Figure $3 \mathrm{C}, \mathrm{D})$, which is consistent with previous reports $[35,36]$. Notably, the miR-339-5p mimic significantly increased Doxorubicin-induced senescence in HCT116 p53+/+ but not in HCT116 p53-/- cells (Figure 3C). Consistently, the miR-339-5p inhibitor significantly inhibited the Doxorubicin-induced senescence in HCT116 p53+/+ but not p53-/- cells (Figure 3D). These results clearly demonstrated that miR-339-5p promotes $\mathrm{p} 53$ function in mediating senescence in colorectal cells in response to stress.

\section{MiR-339-5p inhibits migration and invasion of colorectal tumor cells in a largely p53-dependent manner}

p53 plays an important role in inhibition of tumor metastasis [1, 4, 37]. Cell migration and invasion are two critical steps for tumor cell metastasis [38, 39]. Recently, miR-339-5p was reported to be associated with cancer metastasis and poor prognosis in cancer patients [25-27]. To investigate whether miR-339-5p inhibits tumor cell migration and invasion through the activation of $\mathrm{p} 53$, chamber transwell assays were employed to determine the effects of miR-339-5p on the abilities of cell migration and invasion in HCT116 p53+/+ and p53-/- cells. Compared with HCT116 p53+/+ cells, HCT116 p53-/- cells displayed enhanced abilities of migration and invasion (Figure 4A, B), demonstrating that p53 inhibited migration and invasion of tumor cells, which is consistent with previous reports [4, 37, 40, 41]. Notably, miR-339-5p greatly inhibited migration and invasion of HCT116 p53+/+ cells (Figure 4A, B). The inhibitory effects of miR-339-5p on migration and invasion were much less pronounced in HCT116 p53-/- cells (Figure 4A, B). Furthermore, the miR-339-5p inhibitor significantly promoted the migration and invasion of HCT116 p53+/+ cells, but displayed a much less pronounced effect on migration and invasion in HCT116 p53-/- cells (Figure 4C, D). Taken together, these results indicated that miR-339-5p inhibits migration and invasion of colorectal cancer cells in vitro through its activation of p53 function. 


\section{MiR-339-5p inhibits colorectal tumorigenesis in vivo in a largely p53-dependent manner}

p53 plays a critical role in tumor suppression, and loss of $\mathrm{p} 53$ promotes tumor growth $[1,2]$. To investigate whether miR-339-5p inhibits the colorectal tumorigenesis in vivo through its activation of p53, HCT116 p53+/+ and p53-/- cells were injected (s.c.) into nude mice for xenograft tumorigenesis. When the tumor volume reached $\sim 60 \mathrm{~mm}^{3}$, tumors were injected with miR-con or miR-339-5p mimic (once every 2 days for 6 times). As
A

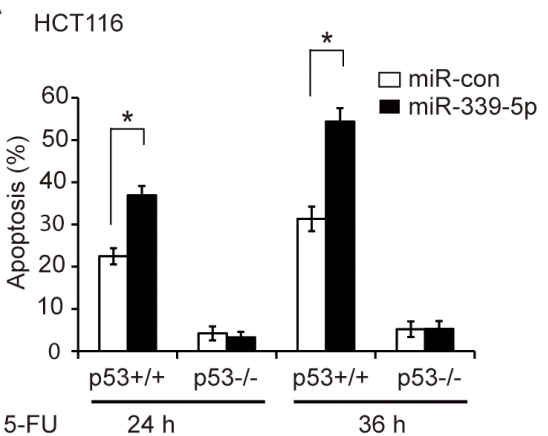

C
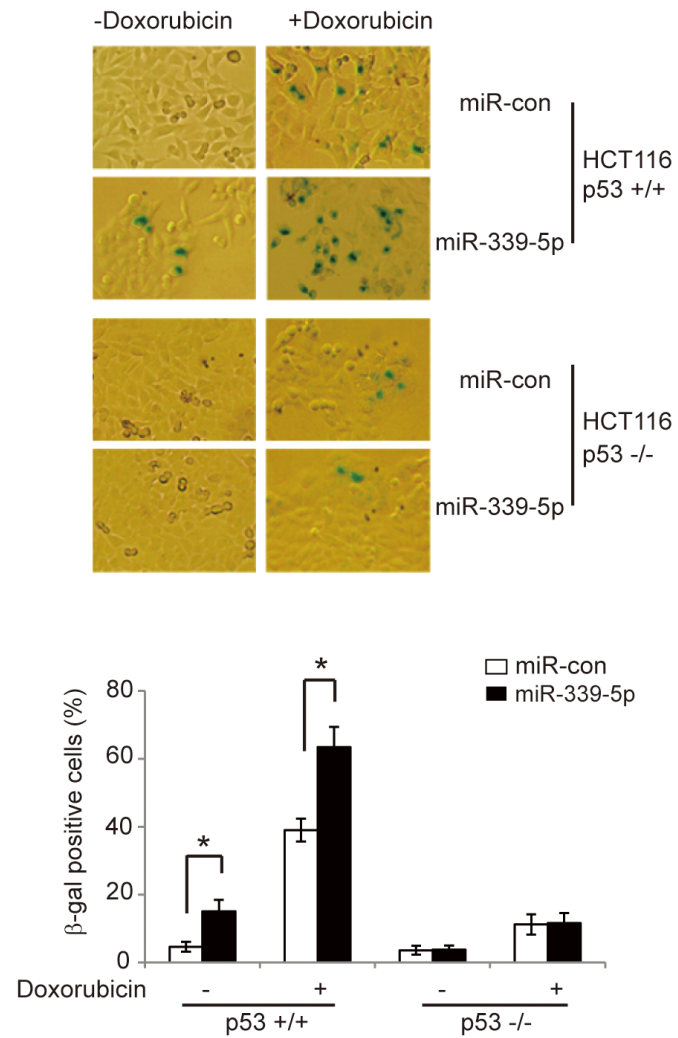

B

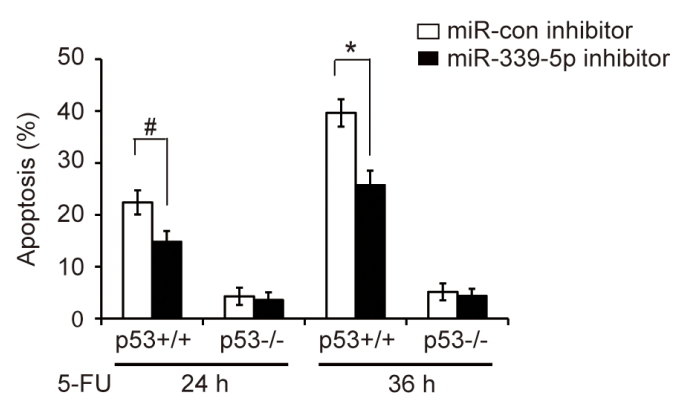

D
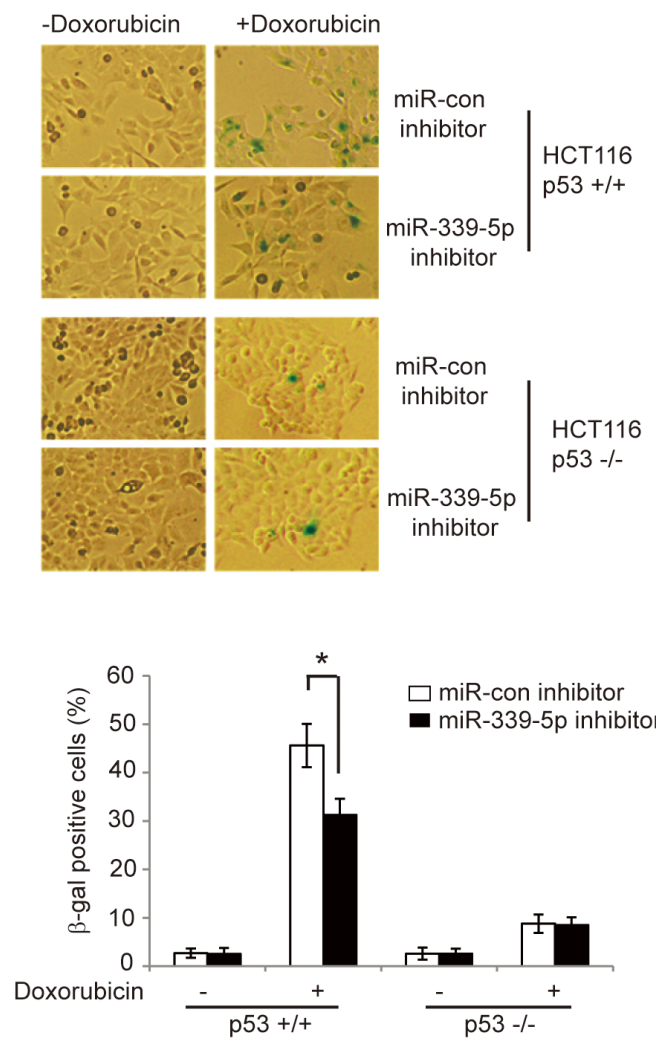

Figure 3: MiR-339-5p enhances p53-mediated apoptosis and senescence in response to stress. (A) MiR-339-5p enhanced p53-mediated apoptosis in HCT116 cells treated with 5-FU. HCT116 p53+/+ and p53-/- cells transfected with miR-339-5p mimic or miR-con were treated with 5-FU $(300 \mu \mathrm{M})$, and apoptosis were measured by Annexin V staining in a flow cytometer at 24 or $36 \mathrm{~h}$ after treatment. (B) The miR-339-5p inhibitor reduced p53-mediated apoptosis in HCT116 cells treated with 5-FU. HCT116 p53+/+ and p53/- cells transfected with the miR-339-5p inhibitor or miR-con inhibitor were treated with 5-FU and analyzed as described in A. (C) MiR339-5p enhanced p53-mediated senescence in HCT116 cells treated with Doxorubicin. HCT116 p53+/+ and p53-/- cells transfected with miR-339-5p mimic or miR-con were treated with $100 \mathrm{nM}$ Doxorubicin for 3 days before cellular senescence was measured by SA- $\beta$-gal staining. (D) The miR-339-5p inhibitor reduced p53-mediated senescence in HCT116 cells treated with Doxorubicin. HCT116 p53+/+ and p53-/- cells transfected with the miR-339-5p inhibitor or miR-con inhibitor were treated with Doxorubicin and analyzed as described in C. In $\mathrm{C}$ and D: The upper panels are represented images of SA- $\beta$-gal staining. In A-D: Data are presented as mean $\pm \mathrm{SD}(\mathrm{n}=3)$. \#: $p<0.05 ;$;: $p<0.01$; two-tailed Student $t$-tests. 
shown in Figure $5 \mathrm{~A} \& \mathrm{~B}, \mathrm{p} 53$ loss promoted the growth of HCT116 tumors as demonstrated by the faster growth rate of HCT116 p53-/- tumors injected with miR-con compared with the HCT116 p53+/+ tumors injected with miR-con, which is consistent with previous reports [18, $42,43]$. Notably, compared with miR-con, miR-339-5p mimic greatly inhibited the growth of HCT116 p53+/+

A

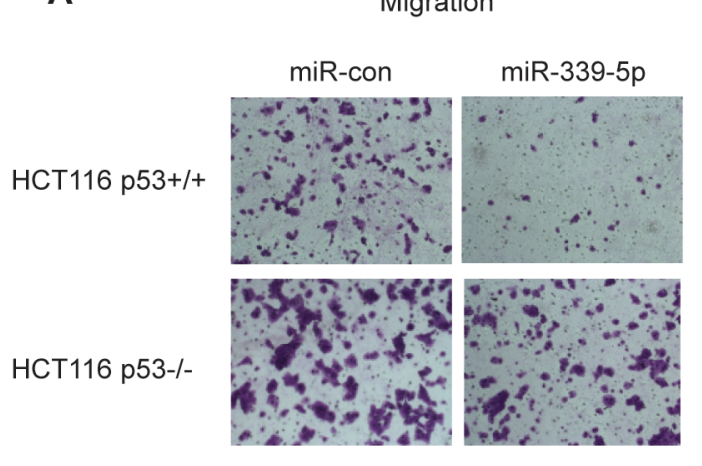

B
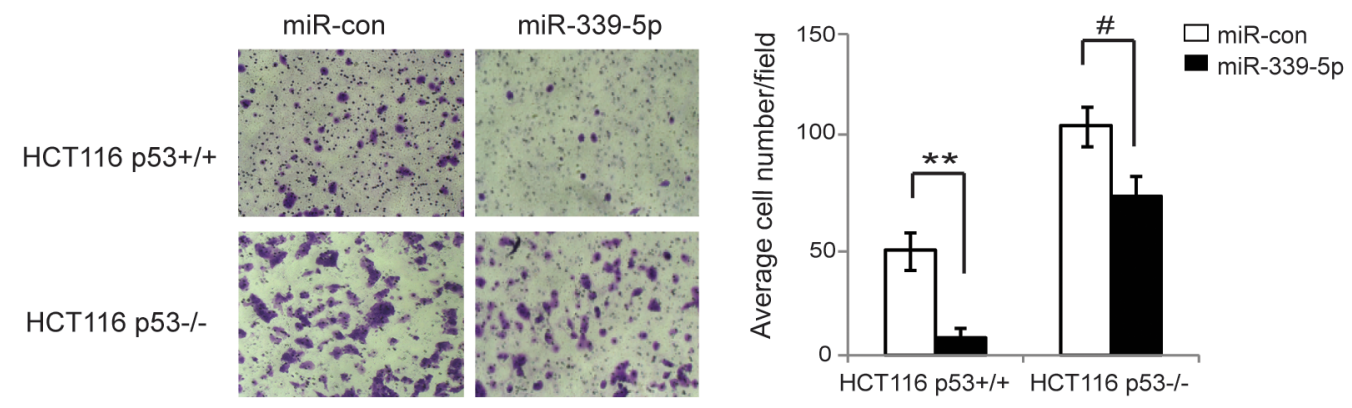

C
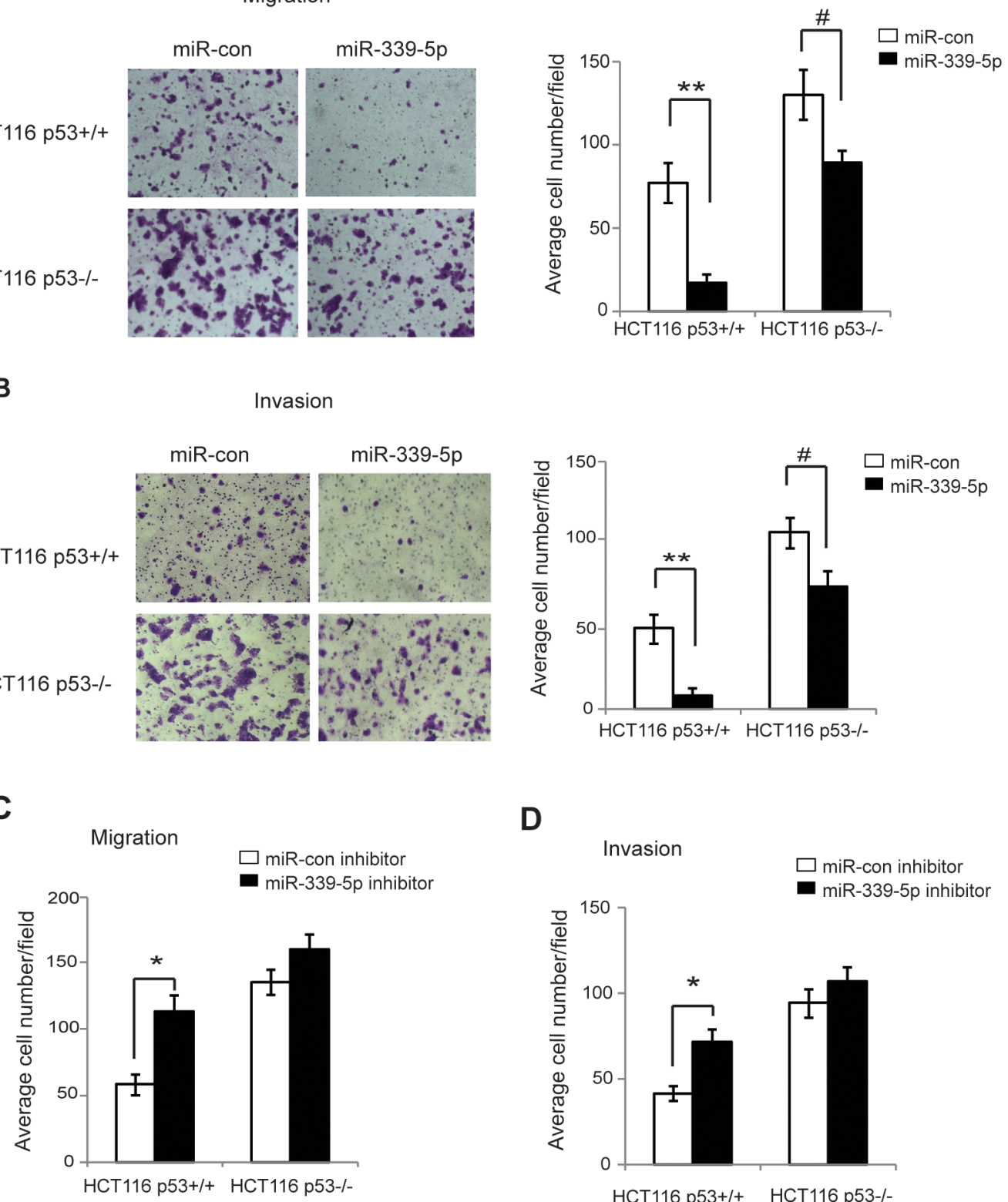

Invasion

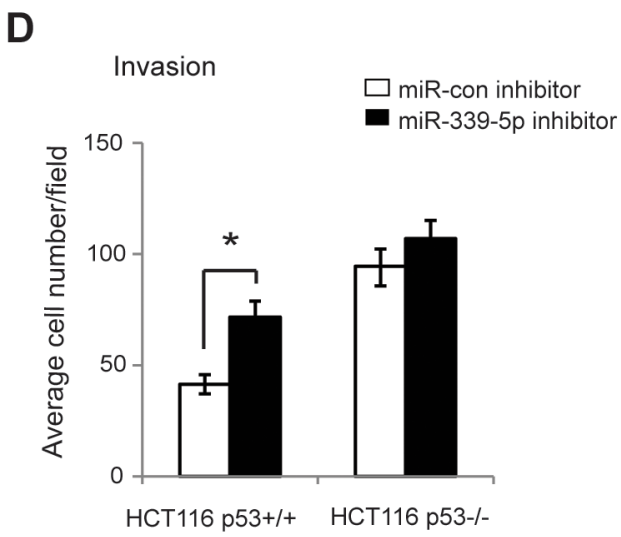

tumors; the tumor volume was reduced by $\sim 6$ fold in HCT116 p53+/+ tumors (Figure 5A, B). This inhibitory effect of miR-339-5p on tumor growth was significantly less pronounced in HCT116 p53-/- tumors; the tumor volume was only reduced by $\sim 2$ fold in HCT116 p53-/tumors (Figure 5A, B). Results from western-blot analysis confirmed that miR-339-5p mimic clearly reduced the

Figure 4: MiR-339-5p inhibits the migration and invasion of colorectal cancer cells in a largely p53-dependent manner. (A) MiR-339-5p inhibited the migration of colorectal cancer cells in a largely p53-dependent manner. HCT116 p53+/+ and p53-/- cells transfected with miR-339-5p mimic or miR-con were seeded into chambers for migration assays. Left panels: Representative images of migrated cells; Right panel: Quantifications of average number of migrated cells per field. (B) MiR-339-5p inhibited the invasion of colorectal cancer cells in a largely p53-dependent manner. HCT116 p53+/+ and p53-/- cells transfected with miR-339-5p mimic or miRcon were seeded into matrigel-coated chambers for invasion assays. Left panels: Representative images of invading cells; Right panel: Quantifications of average number of invading cells per field. (C, D) The miR-339-5p inhibitor promoted the migration (C) and invasion (D) of colorectal cancer cells in a largely p53-dependent manner. HCT116 p53+/+ and p53-/- cells transfected with miR-339-5p inhibitor or miR-con inhibitor were used for migration and invasion assays as described in A and B, respectively. Data are presented as mean $\pm \mathrm{SD}$ $(\mathrm{n}=3) . \#: p<0.05 ; *: p<0.01 ; * *: p<0.001$; two-tailed Student $t$-tests. 
MDM2 protein levels in both HCT116 p53+/+ and p53/- tumors, and increased p53 protein levels in HCT116 p53+/+ tumors (Figure 5C). These results indicated that miR-339-5p inhibits tumor growth in a largely p53-dependent manner in vivo through its direct downregulation of MDM2.

\section{DISCUSSION}

MDM2 plays an important role in tumorgenesis [5, $9,11]$. As an E3 ubiquitin ligase, MDM2 interacts with p53 and mediates the degradation of the p53 protein. MDM2 overexpression and amplification is observed in many types of human cancers, including colorectal cancer, which contributes to tumorigenesis through attenuating p53 function [9-11]. In cells, many signals and factors can regulate the MDM2 protein, including genotoxic stress signals, oncogenic activation, ribosomal stress, chronic stress, neurohormones [11, 44, 45]. Recently, several miRNAs targeting MDM2 have been identified, including miR-192, miR-194, miR-25, miR-32 and miR-661, which revealed that miRNAs are a new component in the
MDM2/p53 signaling pathway [20-22]. In this study, we found that miR-339-5p directly down-regulates MDM2 through binding to the 3'-UTR of MDM2. Through the negative regulation of MDM2, miR-339-5p increases $\mathrm{p} 53$ protein levels and functions, including p53 transcriptional activity, p53-mediated apoptosis and senescence in cells in response to stress. Thus, our results clearly demonstrated that miR-339-5p is an important negative regulator for MDM2 and a positive regulator for p53 in cells.

Recently, several studies reported that the expression of miR-339-5p was decreased in several different types of cancers, including colorectal cancer and breast cancer. Furthermore, lower levels of miR-339-5p were associated with cancer metastasis and poor prognosis in cancer patients [25-27]. These findings strongly suggest a potential role of miR-339-5p in tumor suppression. However, its mechanism is not well understood. In this study, we identified that MDM2 is a direct target for miR-339-5p. Through the negative regulation of MDM2, miR-339-5p increases p53 protein levels and functions. Considering the critical role of p53 in tumor suppression, our findings strongly suggest that activation of p53 is an
A

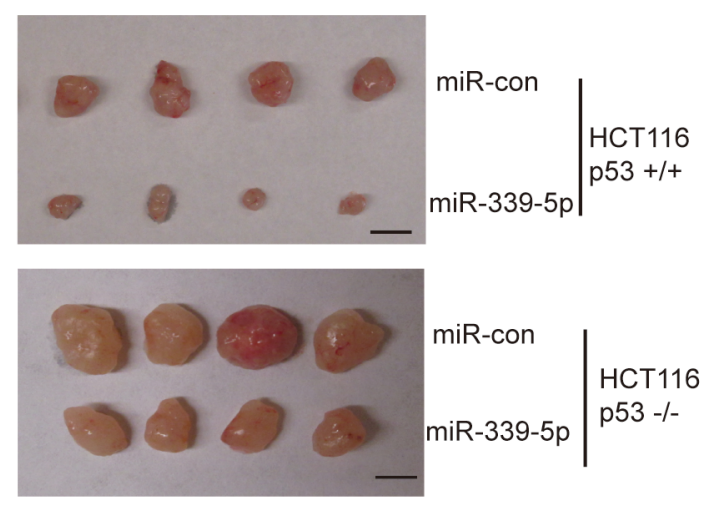

C

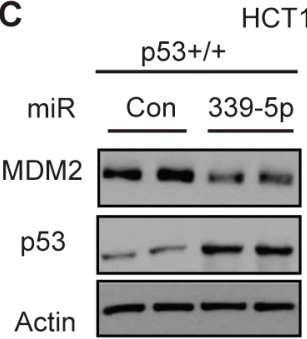

B
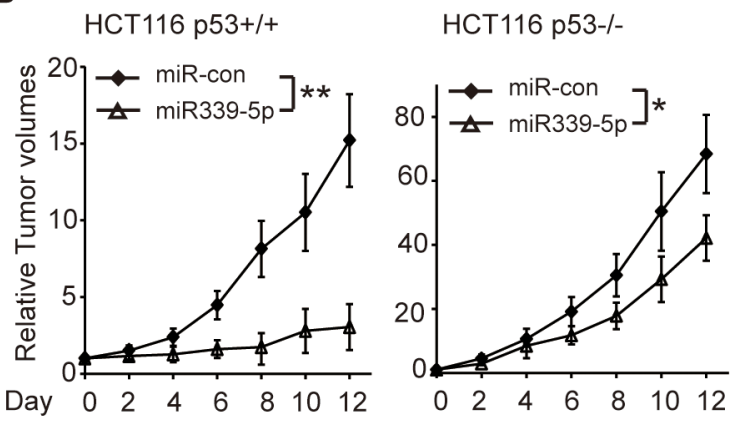

Fold reduction of tumor volumes by miR-339-5p injection

\begin{tabular}{ccc}
\hline HCT116 p53+/+ & HCT116 p53-/- & $p$ \\
\hline $6.3 \pm 1.2$ & $2.1 \pm 0.4$ & $<0.005$ \\
\hline
\end{tabular}

Figure 5: MiR-339-5p inhibits the growth of HCT116 xenograft tumors in vivo in a largely p53-dependent manner. (A, B) MiR-339-5p inhibited the growth of HCT116 xenograft tumors in nude mice in a largely p53-dependent manner. Xenograft tumors were established by s.c. injection of HCT116 p53+/+ and HCT116 p53-/- cells into nude mice. When tumor volumes reached $\sim 60 \mathrm{~mm}^{3}$, tumors were injected with miR-339-5p mimic or miR-con once every two days for 6 times. (A) Representative tumors were photographed at 12 days after the first treatment with miR-339-5p mimic or miR-con. Scale bar: $10 \mathrm{~mm}$. (B) Upper panels: The growth curves of HCT116 p53+/+ and p53-/- tumors after miR-339-5p injection. The relative volumes of the tumors before treatment at day 0 were designated as 1 . Lower panel: The fold reduction of tumor volumes by miR-339-5p injection in both HCT116 p53+/+ and p53-/- tumors. Data are presented as mean $\pm \mathrm{SD}$ ( $\mathrm{n}=12$ for each group). $*: p<0.01$; **: $p<0.001$; two-tailed Student $t$-tests. (C) Injection of miR-339-5p reduced MDM2 protein levels and increased p53 protein levels in HCT116 xenograft tumors. Six tumors from each group were used for western-blot analysis, and very similar results were observed. Represented are 2 tumors from each group. 
important mechanism by which miR-339-5p displays a tumor suppressive function. Consistently, our results in this study showed that miR-339-5p inhibits the migration and invasion of colorectal cancer cells in vitro as well as the growth of colorectal xenograft tumors in mice in a largely p53-dependepnt manner through its repression of MDM2 expression. These results strongly suggest an important role of miR-339-5p in suppression of colorectal tumorigenesis through its regulation of the MDM2/p53 signaling.

Some interesting questions still remain unclear. For instance, the regulation of miR-339-5p in normal and tumor cells is largely unknown. It is unclear whether miR$339-5 \mathrm{p}$ itself can be regulated by stress signals in cells. It is also unclear why the expression of miR-339-5p is frequently down-regulated in different types of tumors. Our results showed that miR-339-5p inhibits tumor cell migration/invasion and xenograft tumor growth in a largely p53-dependenet manner. However, miR-339-5p also displays some p53-independent effects on both tumor cell migration/invasion and tumor growth, although these effects are much less pronounced compared with the p53-dependent effects of miR-339-5p. One miRNA can regulate many different targets [12-14]. Previous studies have identified several other miR-339-5p targets, including BCL-6 and PRL-1 [25, 26]. These identified targets and some additional unidentified targets for miR-339-5p could contribute to the p53-independent activities of miR-339$5 \mathrm{p}$ in tumor suppression. Furthermore, recent studies have reported that MDM2 also exhibits some p53-independent functions $[9,46]$. The repression of MDM2 by miR$339-5 p$ could also lead to the changes of activities and functions of other MDM2-regualted proteins in addition to $\mathrm{p} 53$, which may also contribute to the p53-independent activities of miR-339-5p in tumor suppression. Further studies are needed in the future to address these interesting questions.

In summary, our results in this study demonstrated that miR-339-5p negatively regulates MDM2 in colorectal cancer cells. Through the negative regulation of MDM2, miR-339-5p increases p53 protein levels and functions, including the p53 transcriptional activity and p53mediated apoptosis and senescence in response to stress in colorectal cancer cells. Furthermore, miR-339-5p inhibits the migration and invasion of colorectal cancer cells in vitro and the growth of colorectal xenograft tumors in vivo in a largely p53-dependepnt manner. Thus, our results highlighted an important role of miR-339-5p in suppression of colorectal tumorigenesis through regulating the p53 activity and function.

\section{MATERIALS AND METHODS}

\section{Cells and miRNA transfection}

HCT116 p53+/+, HCT116 p53-/-, RKO p53+/+ and RKO p53-/- cells were generous gifts from Dr. Bert Vogelstein (John Hopkins University). H460 and MCF7 cells were obtained from ATCC. The miRNA mimic and miRNA inhibitor oligonucleotides (Ambion, TX) were transfected into cells using Oligofectamine (Invitrogen) as described [18].

\section{Construction of luciferase reporter vectors and luciferase reporter assays}

The human MDM2 3'-UTR sequences (1580 bp, 38-1617 nt from the start of 3'-UTR) containing three putative miR-339-5p binding sites were amplified by PCR using following two primers: Forward primer 5'ACT AGT TAT AAC CCT AGG AAT TTA GAC AAC C -3' and Reverse primer 5' - AAG CTT ACA TCA TTA CTC CCA TCC CTT AC-3'. These two primers contain HindIII and SpeI recognization sites at the 5' end of the primers, respectively. The PCR products were subcloned into the 3' end of the pMIR-luciferase reporter vector (Ambion) at HindIII and SpeI sites. The mutations of three putative miR-339-5p binding sites were introduced using a Quikchange II XL Site-Directed Mutagenesis Kit (Stratagene/Agilent Technologies). Luciferase reporter assays were performed as previously described [18]. In brief, firefly pMIR-luciferase reporter vectors (100 ng) were transfected into cells in 6-well plates together with miR-339-5p mimic (100 nM) or scrambled miRNA mimic as a negative control by using Lipofectamine 2000. pRL-SV40 vectors (5 ng) that express Renilla luciferase (Promega) were co-transfected to normalize the transfection efficiency. Luciferase activities were measured at $24 \mathrm{~h}$ after transfection by using the Dual Luciferase Assay Kit (Promega).

\section{Western-blot assays}

Standard western-blot assays were used to analyze protein expression as we previously described [47]. Following antibody were used: anti-MDM2 (2A10; generous gift from Dr. Arnold Levine) [48], anti-p53 (FL393, Santa Cruz Biotechonology), anti-p21 (Ab-1, EMD Millipore), anti-actin (\#A5441, Sigma). The band intensity on western-blots was quantified by digitalization of the X-ray film and analyzed with Image J 1.45 s software (NIH) and normalized to Actin. 


\section{Taqman Real-Time PCR Analysis}

Total RNA from cells was prepared by using an RNeasy kit (Qiagen). cDNA was prepared with random primers using TaqMan reverse transcription kit (Applied Biosystems) as we previously described [18]. Gene expression levels were determined by real-time PCR using Taqman PCR master mixture and primers. The expression of genes was normalized to actin gene.

\section{Cellular apoptosis and senescence analysis}

Cellular apoptosis and senescence assays were performed as previously described [49]. For apoptosis analysis, cells were treated with 5 -FU $(300 \mu \mathrm{M})$, and collected at different time points (24 and $36 \mathrm{~h})$ after treatment. Cells were washed with PBS, stained with a Alexa Fluor ${ }^{\circledR} 488$ annexin V/Dead Cell Apoptosis Kit (Life Technologies) before being analyzed in a flow cytometer (Beckman Coulter). For senescence analysis, senescent cells were detected by using a Senescence $\beta$-Galactosidase Staining Kit (Cell Signaling) according to the manufacturer's protocol.

\section{Cell migration and invasion assays}

The transwell systems ( 24 wells, $8 \mu \mathrm{M}$ pore size, BD Biosciences) were used for cell migration and invasion assays as we previously described [50]. For migration assays, $5 \times 10^{4}$ cells in $300 \mu \mathrm{l}$ of serum-free medium were seeded into upper chambers, and the lower chamber was filled with $750 \mu \mathrm{l}$ medium supplemented with $10 \%$ FBS. For invasion assays, transwell membranes were precoated with $50 \mu 1$ Matrigel (BD Biosciences), and $1 \times 10^{5}$ cells were seeded into upper chambers. After cells were cultured at $37{ }^{\circ} \mathrm{C}$ for $24 \mathrm{~h}$ (for migration assays) or 36 $\mathrm{h}$ (for invasion assays), cells in the upper surface of the membrane were removed and cells on the lower surface were fixed with methanol and stained with crystal violet. The number of cell was counted in at least five randomly selected fields under a microscope.

\section{Xenograft tumorigenicity assays}

Xenograft tumorigenicity assays were performed as previously described with the approval of the Institutional Animal Care and Use Committee [47]. HCT116 p53+/+ and HCT116 p53-/- cells $\left(5 \times 10^{6}\right.$ in $0.2 \mathrm{ml}$ PBS $)$ were injected (s.c.) into seven-week-old BALB/c nu/nu male athymic nude mice (Taconic, NY). When the volumes of xenograft tumors reached $\sim 60 \mathrm{~mm}^{3}$, miR-339-5p mimic $(0.5 \mathrm{nmol})$ or scrambled miRNA-con were injected directly into the tumors every two days for 6 times $(n=12$ per group), and mice were sacrificed at the day 12 (the day for the first injection was designated as day 0 ), and tumors were collected for analysis. Tumor volume $=1 / 2($ length $\times$ width $^{2}$ ).

\section{Statistical analysis}

The differences in tumor growth among groups were analyzed for statistical significance by ANOVA, followed by Student's $t$-test using a GraphPad Prism software. All other $P$ values were obtained using two-tailed Student $t$-tests.

\section{Conflict of interest}

The authors declare no conflict of interest.

\section{ACKNOWLEDGEMENTS}

This work was supported by grants from the NIH (1R01CA143204), UMDNJ Foundation and CINJ Foundation (to Z.F.). C.Z. and J. L. were supported by NJCCR postdoctoral fellowships.

\section{REFERENCES}

1. Vousden $\mathrm{KH}$ and Prives C. Blinded by the Light: The Growing Complexity of p53. Cell. 2009; 137(3):413-431.

2. Levine $\mathrm{AJ}$ and Oren $\mathrm{M}$. The first 30 years of $\mathrm{p} 53$ : growing ever more complex. Nat Rev Cancer. 2009; 9(10):749-758.

3. Vogelstein B, Lane D and Levine AJ. Surfing the p53 network. Nature. 2000; 408(6810):307-310.

4. Powell E, Piwnica-Worms D and Piwnica-Worms H. Contribution of p53 to metastasis. Cancer discovery. 2014; 4(4):405-414.

5. Brooks $\mathrm{CL}$ and $\mathrm{Gu}$ W. p53 ubiquitination: $\mathrm{Mdm} 2$ and beyond. Mol Cell. 2006; 21(3):307-315.

6. Harris SL, Gil G, Robins H, Hu W, Hirshfield K, Bond $\mathrm{E}$, Bond $\mathrm{G}$ and Levine AJ. Detection of functional singlenucleotide polymorphisms that affect apoptosis. Proc Natl Acad Sci U S A. 2005; 102(45):16297-16302.

7. Momand J, Zambetti GP, Olson DC, George D and Levine AJ. The mdm-2 oncogene product forms a complex with the p53 protein and inhibits p53-mediated transactivation. Cell. 1992; 69(7):1237-1245.

8. Oliner JD, Kinzler KW, Meltzer PS, George DL and Vogelstein B. Amplification of a gene encoding a p53associated protein in human sarcomas. Nature. 1992; 358(6381):80-83.

9. Manfredi JJ. The Mdm2-p53 relationship evolves: Mdm2 swings both ways as an oncogene and a tumor suppressor. Genes \& development. 2010; 24(15):1580-1589.

10. Momand J, Jung D, Wilczynski S and Niland J. The MDM2 gene amplification database. Nucleic Acids Res. 1998; 
26(15):3453-3459.

11. Wade M, Li YC and Wahl GM. MDM2, MDMX and p53 in oncogenesis and cancer therapy. Nat Rev Cancer. 2013; 13(2):83-96.

12. Pillai RS, Bhattacharyya SN and Filipowicz W. Repression of protein synthesis by miRNAs: how many mechanisms? Trends Cell Biol. 2007; 17(3):118-126.

13. Bartel DP. MicroRNAs: target recognition and regulatory functions. Cell. 2009; 136(2):215-233.

14. Kasinski AL and Slack FJ. Epigenetics and genetics. MicroRNAs en route to the clinic: progress in validating and targeting microRNAs for cancer therapy. Nat Rev Cancer. 2011; 11(12):849-864.

15. Feng Z, Zhang C, Wu R and Hu W. Tumor suppressor p53 meets microRNAs. J Mol Cell Biol. 2011; 3(1):44-50.

16. Jansson MD and Lund AH. MicroRNA and cancer. Mol Oncol. 2012; 6(6):590-610.

17. Hermeking H. MicroRNAs in the p53 network: micromanagement of tumour suppression. Nat Rev Cancer. 2012; 12(9):613-626.

18. Hu W, Chan CS, Wu R, Zhang C, Sun Y, Song JS, Tang LH, Levine AJ and Feng Z. Negative regulation of tumor suppressor p53 by microRNA miR-504. Mol Cell. 2010; 38(5):689-699.

19. Le MT, Teh C, Shyh-Chang N, Xie H, Zhou B, Korzh V, Lodish HF and Lim B. MicroRNA-125b is a novel negative regulator of p53. Genes \& development. 2009; 23(7):862876.

20. Suh SS, Yoo JY, Nuovo GJ, Jeon YJ, Kim S, Lee TJ, Kim T, Bakacs A, Alder H, Kaur B, Aqeilan RI, Pichiorri F and Croce CM. MicroRNAs/TP53 feedback circuitry in glioblastoma multiforme. Proc Natl Acad Sci U S A. 2012; 109(14):5316-5321.

21. Hoffman Y, Bublik DR, Pilpel Y and Oren M. miR-661 downregulates both Mdm2 and Mdm4 to activate p53. Cell Death Differ. 2014; 21(2):302-309.

22. Pichiorri F, Suh SS, Rocci A, De Luca L, Taccioli C, Santhanam R, Zhou W, Benson DM, Jr., Hofmainster C, Alder H, Garofalo M, Di Leva G, Volinia S, Lin HJ, Perrotti D, Kuehl M, et al. Downregulation of p53-inducible microRNAs 192, 194, and 215 impairs the p53/MDM2 autoregulatory loop in multiple myeloma development. Cancer Cell. 2010; 18(4):367-381.

23. Iorio $\mathrm{MV}$ and Croce CM. MicroRNA dysregulation in cancer: diagnostics, monitoring and therapeutics. A comprehensive review. EMBO Mol Med. 2012; 4(3):143159.

24. Lu J, Getz G, Miska EA, Alvarez-Saavedra E, Lamb J, Peck D, Sweet-Cordero A, Ebert BL, Mak RH, Ferrando AA, Downing JR, Jacks T, Horvitz HR and Golub TR. MicroRNA expression profiles classify human cancers. Nature. 2005; 435(7043):834-838.

25. Zhou C, Liu G, Wang L, Lu Y, Yuan L, Zheng L, Chen F, Peng F and Li X. MiR-339-5p regulates the growth, colony formation and metastasis of colorectal cancer cells by targeting PRL-1. PLoS One. 2013; 8(5):e63142.

26. Wu ZS, Wu Q, Wang CQ, Wang XN, Wang Y, Zhao JJ, Mao SS, Zhang GH, Zhang N and Xu XC. MiR-339$5 \mathrm{p}$ inhibits breast cancer cell migration and invasion in vitro and may be a potential biomarker for breast cancer prognosis. BMC Cancer. 2010; 10:542.

27. Li Y, Zhao W, Bao P, Li C, Ma XQ, Li Y and Chen LA. miR-339-5p inhibits cell migration and invasion and may be associated with the tumor-node-metastasis staging and lymph node metastasis of non-small cell lung cancer. Oncol Lett. 2014; 8(2):719-725.

28. Hu W, Feng Z, Teresky AK and Levine AJ. p53 regulates maternal reproduction through LIF. Nature. 2007; 450(7170):721-724.

29. Hu W, Zhang C, Wu R, Sun Y, Levine A and Feng Z. Glutaminase 2, a novel p53 target gene regulating energy metabolism and antioxidant function. Proc Natl Acad Sci U S A. 2010; 107(16):7455-7460.

30. Bunz F, Dutriaux A, Lengauer C, Waldman T, Zhou S, Brown JP, Sedivy JM, Kinzler KW and Vogelstein B. Requirement for p53 and p21 to sustain G2 arrest after DNA damage. Science. 1998; 282(5393):1497-1501.

31. Matoba S, Kang JG, Patino WD, Wragg A, Boehm M, Gavrilova O, Hurley PJ, Bunz F and Hwang PM. p53 regulates mitochondrial respiration. Science. 2006; 312(5780):1650-1653.

32. Feng $\mathrm{Z}$ and Levine AJ. The regulation of energy metabolism and the IGF-1/mTOR pathways by the $\mathrm{p} 53$ protein. Trends Cell Biol. 2010; 20(7):427-434.

33. Hwang PM, Bunz F, Yu J, Rago C, Chan TA, Murphy MP, Kelso GF, Smith RA, Kinzler KW and Vogelstein B. Ferredoxin reductase affects p53-dependent, 5-fluorouracilinduced apoptosis in colorectal cancer cells. Nat Med. 2001; 7(10):1111-1117.

34. O'Connor PM, Jackman J, Bae I, Myers TG, Fan S, Mutoh M, Scudiero DA, Monks A, Sausville EA, Weinstein JN, Friend S, Fornace AJ, Jr. and Kohn KW. Characterization of the p53 tumor suppressor pathway in cell lines of the National Cancer Institute anticancer drug screen and correlations with the growth-inhibitory potency of 123 anticancer agents. Cancer research. 1997; 57(19):42854300 .

35. Elmore LW, Rehder CW, Di X, McChesney PA, JacksonCook CK, Gewirtz DA and Holt SE. Adriamycin-induced senescence in breast tumor cells involves functional p53 and telomere dysfunction. The Journal of biological chemistry. 2002; 277(38):35509-35515.

36. Chang BD, Xuan Y, Broude EV, Zhu H, Schott B, Fang $\mathrm{J}$ and Roninson IB. Role of p53 and p21waf1/cip1 in senescence-like terminal proliferation arrest induced in human tumor cells by chemotherapeutic drugs. Oncogene. 1999; 18(34):4808-4818.

37. Muller PA, Vousden KH and Norman JC. p53 and its 
mutants in tumor cell migration and invasion. J Cell Biol. 2011; 192(2):209-218.

38. Chiang AC and Massague J. Molecular basis of metastasis. N Engl J Med. 2008; 359(26):2814-2823.

39. Chaffer CL and Weinberg RA. A perspective on cancer cell metastasis. Science. 2011; 331(6024):1559-1564.

40. Hwang CI, Matoso A, Corney DC, Flesken-Nikitin A, Korner S, Wang W, Boccaccio C, Thorgeirsson SS, Comoglio PM, Hermeking H and Nikitin AY. Wild-type p53 controls cell motility and invasion by dual regulation of MET expression. Proc Natl Acad Sci U S A. 2011; 108(34):14240-14245.

41. Moskovits N, Kalinkovich A, Bar J, Lapidot T and Oren M. p53 Attenuates cancer cell migration and invasion through repression of SDF-1/CXCL12 expression in stromal fibroblasts. Cancer Res. 2006; 66(22):10671-10676.

42. Yoon CH, Lee ES, Lim DS and Bae YS. PKR, a p53 target gene, plays a crucial role in the tumor-suppressor function of p53. Proc Natl Acad Sci U S A. 2009; 106(19):78527857.

43. Buzzai M, Jones RG, Amaravadi RK, Lum JJ, DeBerardinis RJ, Zhao F, Viollet B and Thompson CB. Systemic treatment with the antidiabetic drug metformin selectively impairs p53-deficient tumor cell growth. Cancer research. 2007; 67(14):6745-6752.

44. Hu W, Feng Z and Levine AJ. The Regulation of Multiple p53 Stress Responses is Mediated through MDM2. Genes Cancer. 2012; 3(3-4):199-208.

45. Perry ME. The regulation of the p53-mediated stress response by MDM2 and MDM4. Cold Spring Harb Perspect Biol. 2010; 2(1):a000968.

46. Zhao $\mathrm{Y}, \mathrm{Yu} \mathrm{H}$ and $\mathrm{Hu} \mathrm{W}$. The regulation of MDM2 oncogene and its impact on human cancers. Acta biochimica et biophysica Sinica. 2014; 46(3):180-189.

47. Zhang C, Liu J, Liang Y, Wu R, Zhao Y, Hong X, Lin M, Yu H, Liu L, Levine AJ, Hu W and Feng Z. Tumourassociated mutant p53 drives the Warburg effect. Nature communications. 2013; 4:2935.

48. Chen J, Wu X, Lin J and Levine AJ. mdm-2 inhibits the G1 arrest and apoptosis functions of the p53 tumor suppressor protein. Mol Cell Biol. 1996; 16(5):2445-2452.

49. Liu J, Zhang C, Wang X, Ly P, Monette X, Belyi V, Young $\mathrm{K}, \mathrm{Hu} \mathrm{W}$, Feng Z. E3 ubiquitin ligase TRIM32 negatively regulates tumor suppressor $\mathrm{p} 53$ to promote tumorigenesis. Cell Death Differ. 2014; [Epub ahead of print].

50. Zheng T, Wang J, Zhao Y, Zhang C, Lin M, Wang X, Yu H, Liu L, Feng Z and Hu W. Spliced MDM2 isoforms promote mutant p53 accumulation and gain-of-function in tumorigenesis. Nature communications. 2013; 4:2996. 